\title{
Review of: "Stress Analysis And Applicability Analysis of Elliptical Head"
}

\author{
Xiangfeng LV
}

Potential competing interests: The author(s) declared that no potential competing interests exist.

Reviewers' comments

This thesis takes the standard elliptical head as the research object, derives the theoretical stress formula of the discontinuous area between the elliptical head and the cylinder based on the deformation continuity equation, and verifies the accuracy of the theoretical stress results based on the experimental research. Force analysis has certain theoretical significance. However, this article is less innovative. The analysis of the theoretical solution and the response of the experimental analysis are relatively routine, which is more common in previous studies. It is recommended that the author resubmit the revision. In addition, the following issues in the paper still need to be discussed with the author:

प1 1 This paper does not summarize the current situation of the theoretical research and experimental research of the head cover. It is recommended that the author summarize the relevant literature in the past 5 years and provide reasonable supplementary explanations.

$\square 2 \square$ This paper calculates the theoretical stress of the discontinuous region based on the continuous return of deformation, and suggests that the author supplement the construction conditions established by the theoretical equation.

[3]In this paper, through the comparison between the theoretical solution and the experimental data, the stress variation characteristics of the elliptical head under certain internal pressure are analyzed. It is suggested that the author supplements the cause of the mutation of the theoretical solution and the experimental data at the measuring point 2 and 4.

$\square 4 \mathrm{I}$ In the end of the paper, the internal pressure of $0.1 \mathrm{MPa}$ was used as an example to analyze the consistency between the theoretical solution and the experimental data. It is recommended that the author supplement the data comparison of other groups to enrich the data basis.

In summary, I suggest that the author revise and resubmit. 